\title{
Argumentation to Represent and Reason over Biological Systems
}

\author{
Adam Wyner ${ }^{1 \star \star}$, Luke Riley ${ }^{1}$, Robert Hoehndorf ${ }^{2}$, and Samuel Croset $^{3}$ \\ 1 Department of Computer Science, University of Liverpool, Liverpool, UK \\ 2 Department of Genetics, University of Cambridge, Cambridge, UK \\ ${ }^{3}$ European Bioinfomatics Institute, Cambridge, UK
}

\begin{abstract}
In systems biology, networks represent components of biological systems and their interactions. It is a challenge to efficiently represent, integrate and analyse the wealth of information that is now being created in biology, where issues concerning consistency arise. As well, the information offers novel methods to explain and explore biological phenomena. To represent and reason with inconsistency as well as provide explanation, we represent a fragment of a biological system and its interactions in terms of a computational model of argument and argumentation schemes. Process pathways are represented in terms of an argumentation scheme, then abstracted into a computational model for evaluation, yielding sets of 'consistent' arguments that represent compatible biological processes. From the arguments, we can extract the corresponding processes. We show how the analysis supports explanation and systematic exploration in a biology network.
\end{abstract}

Keywords: argumentation, systems biology, computational methods

\section{Introduction}

Systems biology is an inter-disciplinary field that emphasizes the analysis of whole biological systems and the interactions occurring within them. Instead of reducing the behavior of biological systems to that of its parts, biological phenomena are studied as components in a network of interrelated processes that span multiple domains and levels of granularity [14. Computational methods in systems biology rely on the construction of models that can predict the behavior of biological systems, the integration of large amounts of data derived from multiple sources, experimental methods and domains as well as the study of networks of interactions between the components of biological systems [13. Biological networks represent components of biological systems and their interactions [3], which have been crucial in the analysis of protein-protein interactions [12], side-effects [15] and human disease [11].

Several large curated knowledge repositories have been created to store information about these interactions 6[10]15, and the application of high-throughput

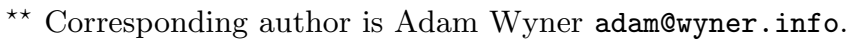


technologies in molecular biology further contributes to the rapid increase of information about interactions that occur in biological systems. It is an ongoing challenge to efficiently represent, integrate and analyze the wealth of information that is now being created in biology. Integration and analysis of data in systems biology is challenging on different levels: first, for curators of scientific data and knowledge bases, it is difficult to identify inconsistencies from source textual materials; second, data repositories may be incomplete, error-prone, or inconsistent; third, combining multiple repositories is difficult and can lead to inconsistencies; and fourth, the wealth of information that is now available requires entirely new analysis methods that can identify explanations and supporting evidence for biological phenomena.

Novel computational representations and implementations that can automatically represent and reason over biological phenomena can facilitate curation of databases, data retrieval, integration of data across multiple domains and levels of granularity, and assemble alternative or competing interpretations for experiment results. Here, we explore the possibility to represent biological systems and their interactions in terms of argumentation theory, a computational model of argument which is used to represent and reason with inconsistency. We will use the example of biochemical pathways to illustrate how components and their interactions in biological systems can be represented using the framework of argumentation theory.

Abstract argumentation frameworks (AFs) are a means to represent and reason with inconsistencies. AFs use graphs of nodes and arcs, where the nodes represent abstract arguments, having no internal structure, and the arcs represent attacks between the arguments [7. Over complex networks of arguments, we can calculate extensions, which are sets of arguments which are mutually compatible, though the intersection of the sets has incompatible arguments. Where arguments are related to component propositions, then extensions are semantic models of a domain. Adding or subtracting arguments (and their corresponding attacks) from an AF gives rise to alternative extensions. Such frameworks have been widely developed to handle non-monotonic reasoning.

However, abstract arguments are not useful for representing instantiated arguments, that is, arguments with some internal structure or content such as in logical syllogisms or in presumptive reasoning argumentation schemes [20]. Such instantiated arguments appear in knowledge bases, which themselves are widespread for many domains. Some efforts have been made to relate abstract to instantiated arguments [1|2|5|19|23; these tend to have domain specific forms. One of the strengths of argumentation schemes is that they provide an 'explanation' or 'justification' of a conclusion; where schemes are chained together, a rich explanation is provided.

In this paper, we develop an argumentation scheme to support reasoning for biomolecular pathways; a reaction in a pathway is represented in terms of propositions in an argument that has premises, exceptions, a rule, and a conclusion, where the inhibitions or perturbations are represented as exceptions. Instantiated schemes or chains of schemes represent arguments. Such arguments can 
stand in attack relations, where the conclusion of one argument is the negation of part of another argument. Where we abstract from the schemes and give the attacks, we can express the network in an argumentation framework and calculate extensions. In this way, we can represent knowledge bases of biomolecular pathways, reason with inconsistency that may arise either between knowledge bases or as knowledge in a domain grows, and explain outcomes. More importantly, argumentation theory provides a novel approach to analyze chains of complex interactions in biological systems, evaluate the consequences of defects in these systems, and possibly provide a model of therapeutic strategies for complex diseases with a molecular basis. While an extensive evaluation of our framework based on real biological data is future work, we exercise the analysis with respect to a small, worked example derived from an existing pathway knowledge base, illustrating how biologically significant questions about interaction networks can be restated as operations in an argumentation framework.

The paper has the following sections. Our materials and queries for biomolecular pathways are indicated in section 2. In section 3, we outline argumentation frameworks and instantiated argumentation. The formal language in which our biomolecular argumentation scheme is expressed is given in section 4 , the language underpins the scheme. The scheme is given in section 5 , then instantiated. Additional instantiations are then shown to represent a fragment of a given biomolecular pathway. In effect, a knowledge base for biomolecular pathways is translated into a format that suits tableau reasoning. The advantage of the instantiated scheme is that it gives specific locations for attack. To exercise the analysis, we abstract from the particulars of the instantiated scheme to represent an $\mathrm{AF}$ and its extensions. Concerning the introduction or removal of particular elements of the AF, we calculate extensions with respect to alternative attacks, which may be interpreted as in silico experiments. We discuss related work in section 6 and future work in 7 .

\section{Systems Biology Background}

The behavior of complex biological entities such as cells and organisms is the result of interacting entities across multiple scales of granularity. For example, the type of proteins that are expressed in a cell will determine the function of the cell through a complex network of interactions such as positive and negative regulations. Depending on which proteins are present in the cell (i.e., expressed and then translated), the functions of cells (and, on a higher level of granularity, tissues and organs) change. Not all proteins are expressed simultaneously; instead, modules of protein interaction networks are stable sets of proteins that usually are expressed together in order to result in stable functioning of a cell. Furthermore, these proteins do not interact randomly, but are based on stable pathways that evolved over time. Pathways are chains of interactions that have been identified as significant because they result in a particular biological function or a product that is crucial for the functioning of a cell. Several pathway databases aim to capture this information. A 'normative' state of a cell such as 
expressed by a pathway or a network of interacting proteins may be disrupted, either pathologically in the case of a disease or disorder, or by the introduction of a drug or another biological agent such as a microRNA (which negatively regulate the transcription of mRNAs). Depending on which of the proteins are present in a cell and how they interact, the physiology of the cell, and subsequently the tissue and organ of which the cell is a part, changes.

Important questions about interaction networks in systems biology include the identification of stable functional modules, i.e., entities and interactions that may occur simultaneously and in parallel without conflict 3. Once we are able to identify such modules, we can investigate the effect of changing the normative behavior of such interaction networks. For example, we can investigate the effect of inhibiting particular interactions or interacting entities, such as when we introduce drugs or regulatory elements such as microRNAs that either selectively inhibit the activity of molecules or disrupt the occurrence of interactions between molecules [4. In a more complex scenario, we may want to identify an entity (e.g., a drug) that, when added to an interaction network, can achieve a desired outcome while at the same time minimizing adverse reactions resulting from this introduction. Ultimately, these operations would be evaluated against experimental data such as gene expression experiments.

Our basic assumption is that argumentation frameworks can not only provide the means to reason with inconsistent knowledge bases, but also provide the means to analyze interactions in biological systems. In particular, we aim to test the hypothesis that some types of interaction networks in biology can be represented as networks of arguments, and that notions such as consistency, rule, premise and attack from argumentation theory correspond to constituents of biological systems and their underlying laws.

\section{Argumentation Frameworks and Instantiated Argumentation}

To represent and reason with the information in the biological pathways data, we represent pathways as instantiated arguments in an argumentation framework, which we present in this section.

An abstract argument framework, as introduced by Dung, 7] is a pair $A F=$ $\langle\mathcal{A}$, attack $\rangle$, where $\mathcal{A}$ is a set of arguments and attack a binary relation on $\mathcal{A}$. A subset $\mathcal{B}$ of $\mathcal{A}$ is said to be conflict-free if no argument in $\mathcal{B}$ attacks another argument in $\mathcal{B}$. $\mathcal{B}$ is said to be admissible if: it is conflict-free; and it defends itself against any attack. For example, suppose arguments $A_{1}$ and $A_{3}$ are in $\mathcal{B}$, some argument $A_{2}$ is in $\mathcal{A}$ but not in $\mathcal{B}$, and $A_{2}$ attacks $A_{1}$; the set $\mathcal{B}$ is admissible when some argument in $\mathcal{B}$, such as $A_{3}$, attacks $A_{2}$. A preferred extension is then a maximal (with respect to set inclusion) admissible set. Several other types of extensions are defined, but they are not used in our model.

Dung's arguments are entirely abstract, with no features other than the attack relation. In order to enable some content to be given to the arguments, 
a refinement of Dung's abstract approach, which provides some structure for arguments, was developed in the ASPIC framework [19].

This framework assumes an unspecified logical language and knowledge base, which may include facts, strict rules, and defeasible rules; it defines arguments $\mathrm{A}_{i}$ as inference trees formed by applying inference rules (which may be either strict or defeasible) to a knowledge base: the nature of the inference rules is also unspecified, though explicitly represented in the arguments.

We represent entailment in strict rules with $\stackrel{s}{\rightarrow}$ and in defeasible rules with $\stackrel{d}{\rightarrow}$. An argument thus comprises a non-leaf node in the tree (the conclusion of the argument) together with the children of that node (the premises of the argument) and the rule from premises to conclusion. Leaf nodes are facts in the knowledge base. The conclusion of argument $\mathrm{A}_{i}$ can be the premise of some other argument $\mathrm{A}_{j}$, allowing us to chain arguments together. By and large, the conclusions and premises of arguments are literals (atomic formulae or their negations), where we use propositional negation, e.g. if we have atomic formula $p$, the negation $\neg p$ is a literal. We can also have expressions of rules and their negations. It is inconsistent to have a literal or rule and its negation.

Arguments can be presented as tableau. Following [19], a strict argument, such as that labeled (A1), with premises $\mathrm{P} 2$ and a strict rule $[P 2 \stackrel{s}{\rightarrow} P 1]$, and conclusion P1 appears as Strict Modus Ponens:

$$
\frac{P 2,[P 2 \stackrel{s}{\rightarrow} P 1]}{P 1}
$$

While a defeasible argument (A2) with premise $\mathrm{P} 4$, defeasible rule $[P 4 \stackrel{d}{\rightarrow} P 5]$, and conclusion P5 appears as Defeasible Modus Ponens:

$$
\frac{P 4,[P 4 \stackrel{d}{\rightarrow} P 5]}{P 5}(A 2)
$$

An argument can appear as a more extended tree, where sub-arguments of the larger argument may be strict or defeasible. A strict argument may have only sub-arguments which themselves are strict, otherwise it is a defeasible argument. For example, an argument (A3) with conclusion P11 has a defeasible intermediate argument (A4) with conclusion P9, so (A3) must then be an instance of a defeasible argumentation reasoning pattern:

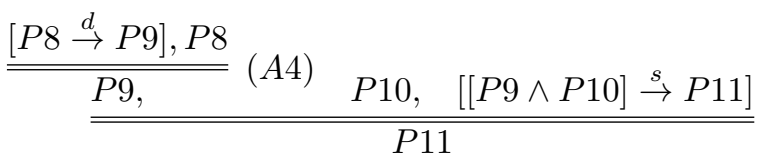

As this example shows, we can have complex arguments in which strict and defeasible arguments appear as intermediate arguments.

The notion of an argument as an inference tree leads to two ways of attacking, rebuttal and undermining, an argument 4

4 The literature on argumentation is more complex and diverse than presented here [19], but the simplification suits our current purposes. 
- An argument $A_{i}$ rebuts an argument $A_{j}$ if the conclusion of $A_{i}$ is $\phi$ and the conclusion of $A_{j}$ is $\neg \phi$; and

- An argument $A_{i}$ undermines an argument $A_{j}$ if the conclusion of $A_{i}$ is $\neg \phi$ and a premise of $A_{j}$ is $\phi$.

For instance, A5 rebuts A1, and A6 undermines A2. As rebuttal is a symmetric attack (unlike undercutting or undermining), we also have A1 rebuts A5:

$$
\begin{gathered}
\frac{P 14,[P 14 \stackrel{d}{\rightarrow} \neg P 1]}{\neg P 1} \\
\frac{P 15,[P 15 \stackrel{d}{\rightarrow} \neg P 4]}{\neg P 4}
\end{gathered}
$$

We can abstract from the structure of the arguments and identify the AF defined by the attack relations among the arguments, for example, where $\mathrm{AF}$ $=\langle\{A 1, A 2, A 3, A 5, A 6\},\{\operatorname{att}(A 5, A 1), \operatorname{att}(A 1, A 5), \operatorname{att}(A 6, A 2)\}\rangle$, then the preferred extensions are: $\{\mathrm{A} 3, \mathrm{~A} 5, \mathrm{~A} 6\}$ and $\{\mathrm{A} 1, \mathrm{~A} 3, \mathrm{~A} 6\}$; nothing attacks $\mathrm{A} 3$ or $\mathrm{A} 6$, and $\mathrm{A} 1$ and $\mathrm{A} 5$ attack one another, so each extension contains one.

Concerning complexity, when an AF has no cycles, the complexity of computing the preferred extension takes time linear to the number of arguments [8]. Preliminary analysis of our working example in section 5.1 suggests that a significant portion of graphs do not produce cycles.

The strength of the approach is that we can clearly and systematically move between the instantiated and abstract arguments and their attack relations; once abstracted, the internal contents of the arguments and their specific relationships are not relevant to calculating extensions, simplifying the reasoning. Furthermore, once we have the extensions, we can then recover the content of the arguments, yielding sets of propositions which are consistent. ASPIC in [19] has a range of other components, though these are not clearly relevant for our purposes.

To this point, we have only represented propositional variables, e.g. P1,.., P15. However, to use the instantiated arguments, we need a knowledge base that represents the information in our domain. Typically, these appear in the form of argumentation schemes [20], which are sterotypical, defeasible reasoning patterns. There are is very large range of such patterns, including Slippery Slope, Ad Homenim, and Practical Reasoning 21. We can introduce domain specific schemes such as has been proposed for legal case-based reasoning [23. For our purpose, the knowledge base should represent schemes for Biomolecular Pathways. Where we represent biological information as argumentation schemes and define attacks between schemes, we can create abstract argumentation frameworks, which would allow us to reason with inconsistent data. 


\section{Biomolecular Action-based Alternating Transition System}

To reconstruct the pathways as argumentation graphs, we express them in a language along the lines of an Action-based Alternating Transition System (AATS) that is designed to represent multi-agent systems [22. The system provides an abstract specification of sets of objects and functions. Instantiated models are defined with respect to the system, where the model satisfies axioms which are the constraints within and between components of the model as well as the incompatibilities among the objects in the components; in other words, constraints and incompatibilities provide an underlying structure to the model, which is then instantiated with particulars. In turn, argumentation schemes are instantiated with respect to the model. We present a derived structure, the Biomolecular Action-based Alternating Transition System (BAATS). From this, we could provide the axioms, a model, and generate the logical space of arguments and the attack graph over which we calculate the extensions, testing the underlying model. This is essentially the approach taken in [1].

A BAATS is a 6 -tuple $S=\langle Q, A c, \rho, \tau, \Phi, \pi\rangle$. Unlike the AATS, there are no autonomous agents, the association of actions and autonomous agents do not hold, and there are no joint actions. Propositions are associated with whether or not a biomolecule holds (in the relevant activatable context).

- $Q$ is a finite, non-empty set of states $\left\{q_{1}, \ldots, q_{n}\right\}$;

- $A c$ is a finite, non-empty set of interactions $\left\{\alpha_{1}, \ldots, \alpha_{n}\right\}$;

$-\rho: A c \rightarrow 2^{Q}$ is an action pre-condition function, which for each interaction $\alpha \in A c$ defines the set of states $\rho(\alpha)$ from which $\alpha$ may be executed;

$-\tau: Q \times A c \rightarrow Q$ is a partial system transition function, which defines the state $\tau(q, \alpha)$, that results by the performance of $\alpha$ from the state $q$, where $\mathrm{q} \in Q$ and $\alpha \in A c$. The function is partial as not all interactions can occur in every state;

- $\Phi$ is a finite, non-empty set of atomic propositions, associated with whether or not a particular biomolecule holds in the current state and location of the biological system;

$-\pi: Q \rightarrow 2^{\Phi}$ is an interpretation function, which gives the set of primitive propositions included in each state: if $p \in \pi(q)$, then this means that the propositional variable $p$ is satisfied (equivalently, true) in state $q$, if $\neg p$ $\in \pi(q)$, then this means that the propositional variable $p$ is not-satisfied (equivalently, false) in state $q$.

Next we turn to the specification of the scheme.

\section{Biomolecular Argumentation Scheme}

We express a biomolecular argumentation scheme (BAS) in terms of the BAATS. In the pathways we are considering, Regulation of nuclear SMAD2/3 signaling 5

\footnotetext{
${ }^{5}$ See Pathway Interaction Database: http://pid.nci.nih.gov/
} 
we take the positive (green arrow), neutral (black arrow), and negative (red arrow) regulators to be premises of a scheme connected to a rule (that is left implicit), which denotes the biomolecular process; the conclusion of the rule is the result of the process given the regulators and is most often a biomolecule. The negative (red) regulators are constraints on the application of a rule, represented in the argumentation scheme as exceptions, meaning that where the exception holds (i.e. true that the literal does not hold), the rule can be applied; where the exception does not hold (i.e. true that the literal does hold), the rule cannot be applied. We do not comment here on attacks on multistate biological processes. Simply put, the reactions in a pathway are recoded as propositions that have assigned roles (e.g. premise, exception, conclusion) in an argumentation scheme ${ }^{6}$

In the following BAS, we provide an argumentation scheme in the language of the BAATS. The schemes could also be presented in tableau format, where we have defeasible rules. The premises of the scheme model the biological process and its conditions (regulators), while the conclusion represents the output of the process given that the conditions hold. The rule we leave implicit. The negative regulator is represented as an exception, which, if present, does not allow us to draw the conclusion. The main justification for the fine-grainedness of the scheme is that we want each proposition in the scheme (premises, rule, and conclusion) to represent specific 'attackable' elements. As we have more than one of each sort of regulator, we distinguish them with superscripts; the premises of each interaction are subscripted to the interaction. The premises co-occur in the same state before the application of the process.

\section{BAS}

1. $\alpha$ : A single step biological process.

2. $\iota_{\alpha}$ : Neutral regulator with respect to $\alpha$, those biomolecular elements with a neutral edge to $\alpha$.

3. $\phi_{\alpha}$ : Positive regulator with respect to $\alpha$, those biomolecular elements with a positive edge to $\alpha$.

4. $\neg \rho_{\alpha}$ : Negative regulator with respect to $\alpha$, those biomolecular elements with a negative edge to $\alpha$.

5. $\tau\left(q_{x}, \alpha\right)=q_{y}$ : The state transition given by $\alpha$, where the presumption is that $\iota_{\alpha}$ and $\phi_{\alpha}$ regulators hold in $q_{x}$, but $\rho_{\alpha}$ does not.

6. Therefore, $\sigma \in q_{y}$ : The result biomolecular element, where the presumption is that the element $\sigma$ holds in the state $q_{y}$ that results after $\alpha$.

In addition to a complex scheme of premises, rule, and conclusion, we assume an Assertion Argument, meaning that literals can be asserted to be true without the need of premises or a rule. In Logic Programming, these are rules that have a head, but no body. In a biomedical domain, an assertion might simply be adding a drug or biomolecular to an existing process.

While we have maintained reference to a dynamic aspect of the processes, when it comes to evaluating the arguments in an abstract argumentation framework, we abstract over the temporal aspect to view the processes statically, as

\footnotetext{
${ }^{6}$ The image in Figure 1 is in greyscale.
} 
co-occuring atemporally since, for the purposes of evaluation, all that matters is the attack relation between arguments. Yet, for the determination of attacks between arguments, we must consider temporality - the attacking element and the attacked element must co-occur. Formally and in an implementation, this is addressed by unification of variables, including a temporal variable.

\subsection{An Example}

For our purposes, we take the Pathway Interaction Database (PID) graph Regulation of nuclear SMAD2/3 signaling as our model which satisfies the biomolecular constraints, presuming the constraints can be defined. It is of interest since there are several negative regulators. First we provide a single instantiated argumentation scheme, then several instantiated schemes with attacks.

From the PID graph, we select the pathway in Figure 1 (modified from the PID graph for clarity). The instantiated argument is referenced as pid_i_200106; in the following instantiations, the prefix pid_i stands for Pathway Interaction Database-Interaction-ID, while pid_m stands for Pathway Interaction DatabaseMolecule-ID, where the ID is found in the graphs associated OWL file via the BioPAX link in the PID. We index premise elements with the process to distinguish between several schemes. The premise names are taken directly, without modification, from the Regulation of nuclear SMAD2/3 signaling graph.

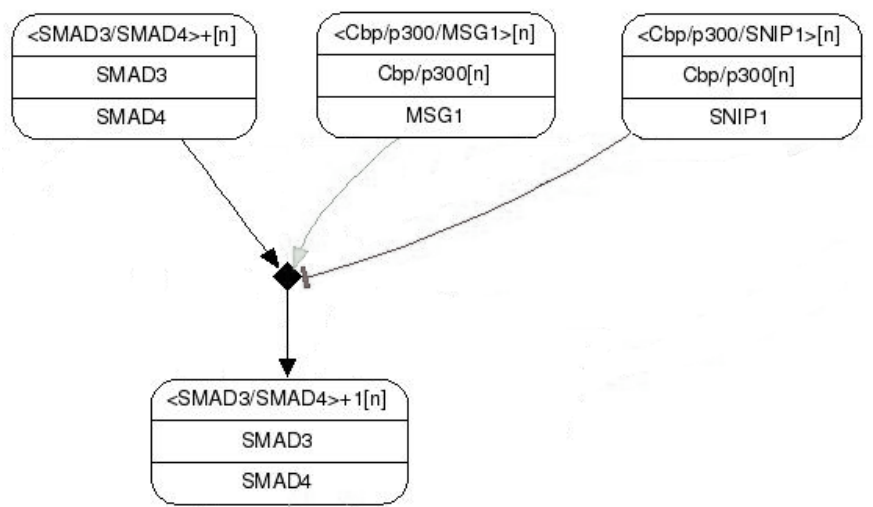

Fig. 1. Biomolecular Pathway Sample 1, where the light grey pointed arrow represents the positive regulator, the black pointed arrow represents the neutral regulator, and the flat-tipped arrow represents the negative regulator.

Argument for $<S M A D 3 / S M A D 4>+1[n]$; pid_i_200106

1. $\alpha^{k}$

2. $\iota_{\alpha^{k}}=<S M A D 3 / S M A D 4>+[n]$

3. $\phi_{\alpha^{k}}=<C b p / p 300 / M S G 1>[n]$

4. $\neg \rho_{\alpha^{k}}=\neg(<C b p / p 300 / S N I P 1>[n])$

5. $\tau\left(q_{x}, \alpha^{k}\right)=q_{y}$

6. Therefore, $<S M A D 3 / S M A D 4>+1[n]$ holds in $q_{y}$

To illustrate attacks between arguments, we instantiate the scheme several times (including pid_i_200106) using those portions of the Regulation of nuclear SMAD2/3 signaling graph that contain negative regulators. 
Argument for $<S M A D 3 / S M A D 4>+1[n]$; pid_i_200104

1. $\alpha^{j}$

2. $\iota_{\alpha^{j}}=<S M A D 3 / S M A D 4>+[n]$

3. $\phi_{\alpha^{j}}^{1}=C b p / p 300[n]$

4. $\phi_{\alpha^{j}}^{2}=P C A F[n]$

5. $\tau\left(q_{x}, \alpha^{j}\right)=q_{y}$

6. Therefore, $<S M A D 3 / S M A D 4>+1[n]$ holds in $q_{y}$

Argument for $<S M A D 3 / S M A D 4 / G R>+[n]$; pid_i_200096

1. $\alpha^{l}$

2. $\iota_{\alpha^{l}}^{1}=G R$

3. $\iota_{\alpha^{l}}^{2}=<S M A D 3 / S M A D 4>+1[n]$

4. $\tau\left(q_{x}, \alpha^{l}\right)=q_{y}$

5. Therefore, $<S M A D 3 / S M A D 4 / G R>+[n]$ holds in $q_{y}$

\section{Argument for $G S C$; pid_i_200037}

1. $\alpha^{m}$

2. $\phi_{\alpha^{m}}=<S M A D 2 / S M A D 2 / S M A D 4 / F O X H 1>+[n]$

3. $\neg \rho_{\alpha^{m}}=\neg(<S M A D 3 / S M A D 4>+1[n])$

4. $\tau\left(q_{x}, \alpha^{m}\right)=q_{y}$

5. Therefore, $G S C$ holds in $q_{y}$

We can introduce assertions about molecules into the representation, e.g.:

Assertion Argument for $<C b p / p 300[n] / S N I P 1>[n]$; pid_m_204265

1. Therefore, $<C b p / p 300[n] / S N I P 1>[n]$ holds in $q_{x}$

\subsection{An Abstract Argumentation Framework}

To give some results for our system fragment, we consider the inter-relations between the instantiated schemes. Where we have undermining or rebuttal, presumptive conclusions do not follow. In particular, note that the conclusions of pid_i_200104 and pid_i_200106 make the exception of pid_i_200037 false, rendering the process of $\mathbf{p i d} \_\mathbf{i} \_\mathbf{2 0 0 0 3 7}$ inapplicable; this means that where the processes of pid_i_200104 and pid_i_200106 apply, the outcome of pid_i200037 does not, presumably, hold as a result. It is in this way that the processes are expressed as arguments in attack relations. Each instantiation of a BAS can be taken as an abstract argument in an argumentation framework. And where one instantiation undermines or rebuts another instantiation, we interprete this as attack between one argument and another. For clarity, we consider three different examples of the analysis: first, we evaluate just the arguments represented in section 5.1 then, we add perturbating arguments; and finally, we make use of an inconsistent knowledge base and related arguments. At each point, we illustrate the impact of assumptions on the relevant extensions.

For our first example of an abstract argumentation framework, we do not consider perturbating arguments; we have the following (where we have not represented the assertion of pid_m_204265): 


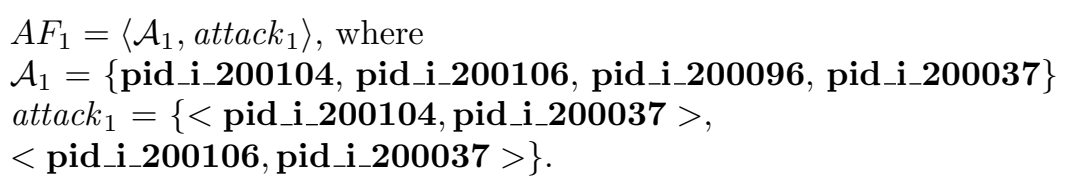

Figure 2 is a graphical representation of $A F_{1}$. Each node is an abstract representation of an instantiation of the BAS (the labels refer to the PID IDs) and the arcs between the nodes represent attacks between the arguments. For discussion below, we have introduced several arguments indicated with the variables, $\mathbf{x}, \mathbf{y}$, and $\mathbf{z}$, that attack other arguments (as indicated). These can be read as arguments for conclusions, e.g. an argument for $x$, an argument for $y$, and an argument for $z$, and the negative conclusions.

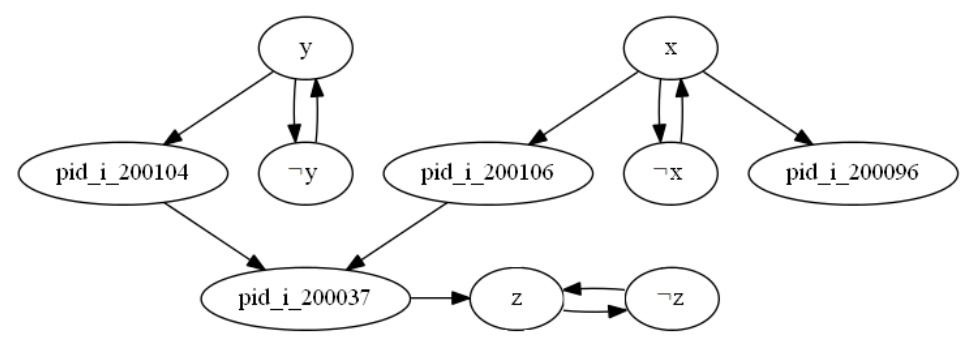

Fig. 2. Biomolecular Pathway Sample 2

Before we determine extensions, we ignore (for the moment) the variable arguments and assume that the premises of attacking arguments in $A F_{1}$ are all true (alternatively, have all been asserted). In this case, the preferred extension is: $P E_{1}=\{$ pid_i_200104, pid_i_200106, pid_i_200096 $\}$, since pid_i_200037 is attacked and not defended by any argument.

For our second example, we consider perturbating arguments. In our analysis, we can represent the action of particular drugs or microRNA (biological perturbating agents) on a given biological system (with instantiated arguments for $\mathbf{x}$ and $\mathbf{y}$ ) as well as the effect of an outcome of a given system on other systems (with an instantiated argument for $\mathbf{z}$ ). For the moment, we ignore the arguments $\neg \mathbf{x}, \neg \mathbf{y}$, and $\neg \mathbf{z}$. We represent perturbating biological agents with instantiated BASs (and so substitute instantiated arguments for the variables $\mathbf{x}$ and $\mathbf{y}$ ), where the conclusion of the argument is the particular perturbating biological agent; this conclusion is the negation of a premise (or conclusion) of some other instantiated BAS; thus, as exemplified above, one argument attacks another argument. Once instantiated, such arguments perturb the system and change its overall state, giving alternative preferred extensions, thus showing the logical effects of drugs in a biological system. In the context of our framework, the extensions capture the resulting state of the system. It is, therefore, possible to carry out in silico experiments which change the output of the overall process model, where different extensions are the result of a drug attack on a set of biological arguments. Alternatively, we may consider the impact of the 
output of a given biological system on other systems; for example, where $\mathbf{z}$ represents an argument for a desirable or at risk clinical end-point, the outcome of pid_i_200037 may (or may not) perturb $\mathbf{z}$, depending on the other arguments and attacks of the biological system. In this way, we can add or subtract elements, noting the overall effect on the extension and making it apparent what sorts of side-effects may arise from different combinations of elements.

To show a sample of this reasoning, we look for relevant elements from PID. Suppose $\mathrm{x}$ is the assertion associated with pid_m_204265, which attacks the exception premise of pid_i_200106 and pid_i_200096; we presume that pid_i_200104 is not attacked.

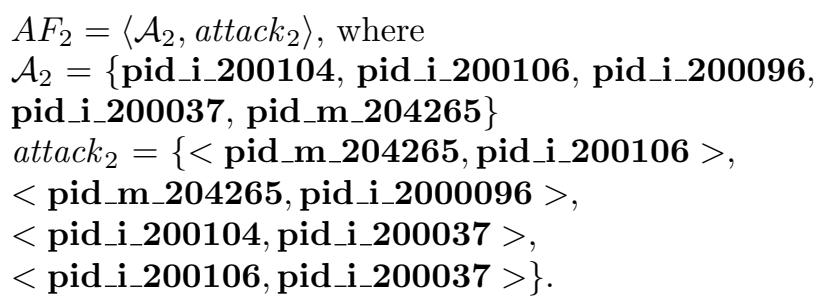

Here, we have: $P E_{2}=\{$ pid_m_204265, pid_i_200104\} .

Alternatively, we can search for a value of $\mathbf{y}$, which would be a biomolecule or process that attacks pid_i_200104, by making one of the premises false; we presume there are no attackers on pid_i_200106 and pid_i_200096. For example, we can search for inhibitors of either $C b p / p 300[n]$ or $P C A F[n]$, which appears to be microRNA miR-181a/b 24. In such an instance, the preferred extension is $P E_{3}=\{\mathbf{m i R}-\mathbf{1 8 1} \mathbf{a} / \mathbf{b}$, pid_i_200106, pid_i_200096 $\}$. When we have both pid_m_204265 and the $\mathbf{m i R}-\mathbf{1 8 1} \mathbf{a} / \mathbf{b}$, then we have $P E_{4}=\{$ pid_m_204265, miR-181a/b, pid_i_200037\}. By the same token, where pid_i_200037 holds, it may serve as an attack on some process such as the variable $\mathrm{z}$ in Figure 2 where the conclusion of pid_i_200037 is the negation of a premise of $\mathrm{z}$.

Finally, we consider all the argument nodes in Figure 2, which represents a knowledge base with inconsistent information. The arguments and attacks can be read off the graph itself. Depending on evaluations of arguments for $\mathbf{x}, \mathbf{y}$, and their negations, alternative extensions are generated as consequences of the attack relations. For instance, supposing $\neg \mathbf{y}$ and $\mathbf{x}$ hold, then we have $P E_{5}=$ $\left\{\neg \mathbf{y}, \mathbf{x}, \mathbf{p i d} \_\mathbf{i}_{-} \mathbf{2 0 0 1 0 4}, \mathbf{z}\right\}$ and $P E_{6}=\left\{\neg \mathbf{y}, \mathbf{x}, \mathbf{p i d} \_\mathbf{i} \_200104, \neg \mathbf{z}\right\}$; we see that given both $\neg \mathbf{y}$ and $\mathbf{x}$, the system is indeterminate with respect to $\mathbf{z}$. On the other hand, where $\mathbf{x}$ and $\mathbf{y}$ holds we have a determinate result for $\neg \mathbf{z}: P E_{7}=$ $\{\mathbf{x}, \mathbf{y}$, pid_i_200037, $\neg \mathbf{z}\}$. Finally, where $\mathbf{y}$ and $\neg \mathbf{x}$ both hold (or where $\neg \mathbf{y}$ and $\neg \mathbf{x}$ both hold), $\mathbf{z}$ is again indeterminate.

The analysis we have presented provides some explanatory power in the sense that we can justify what appears in the extension according to what arguments are attacked or attacking, reasoning backwards through the chains of attack relations. Moreover, extracting the propositional content of the arguments, we can understand the biological terms of the explanation.

Exercising this small fragment shows how argumentation schemes and argumentation frameworks can be used to provide complex explanations for biological 
phenomena, to reason systematically about systems with logical inconsistencies to yield consistent sets of arguments (and the propositions they contain), which can be used to represent states of a biological system, and finally to explore the processes for their interconnections.

\section{Related Work}

In a series of papers, [18, [16], and [17] present an approach to and implementation of argumentation concerning biomolecular pathways. In terms of general subject area and the application of argumentation, their work and that presented here are very closely related. However, the works take different but highly complementary approaches to what is argued about, which is reflected in the sorts of schemes that are deployed. While we focus on an argumentation scheme most like Practical Reasoning in the sense that it is entirely about actions, the work of 17. focuses on Expert Testimony, where experts are called to present their conclusions and counter-conclusions concerning the representation of information in a database. 17. does not take statements in a database as given, but rather aim to identify contradictory statements and large consistent subsets. In our work, we assume the statements in a database as given and investigate the biological consequences of these statements. As a result, [17/18 identify "conflicting information presented by an online biological database", while we aim to identify stable modules of biological interaction networks with certain biological properties. It is a viable area of future research to investigate the relation between both levels of argumentation about biological phenomena.

Another large field of study related to our work is biological network analysis [3] and the use of biological networks in the personalized treatment of disease [4]. In each case, a crucial step is the identification of stable modules in interaction networks that are responsible for physiological processes, which correspond to pathologically abnormal functioning in the case of disease or which determine an organism's response to drugs or environmental factors. While the identification of topological and functional modules in network biology is commonly based on network clustering algorithms that break these networks down into modules of different sizes, depending on the parameters used in the clustering [3], we can identify modules based on global properties governing biological interactions. It is subject to future research to identify to which degree modules identified through our approach correspond to the modules that are traditionally identified in network biology.

\section{Next Steps}

The work will be developed in several directions. First, we will evaluate the accuracy of the representation and reasoning against further data; that is, are the extensions we provide consistent with experimental data and can an implementation handle data on a large scale? To assist in this evaluation over scaled up 
data, it will be necessary to implement a translation from databases into instantiated BASs, to determine their attack relations, and to calculate extensions. In principle, the first step is relatively straightforward, given the structure of the DB and the BAS. Furthermore, the third step already has successful implementations, where arguments and attacks are given, e.g. ASPARTIX [9]. Of more importance is the determination of attack relations given instantiated BASs; this can be addressed so long as strings that are used to represent biomolecules or mRNA are expressed consistently and in an appropriate literal form such that we can search for a string and its negation-prefixed form. Large scale evaluation of this work will have to wait till these issues are fully addressed. A second line of development could be to introduce some preferential information whereby attacks succeed or fail dependent on some additional aspect of the process. A third line of development would be to relax the assumption that attacks are, even if successful, entirely successful. In this approach, we would have attacks that introduce degrees of success somewhat along the lines as discussed in fuzzy logic. This might more realistically model complex biomolecular processes. Finally, it may be interesting and relevant to introduce the temporal element in reasoning in AFs.

\section{Acknowledgements}

Adam Wyner appreciates support from the FP7 IMPACT Project, grant number 247228. Luke Riley is grateful for support from the EPSRC. Robert Hoehndorf is thankful for funding from the FP7 RICORDO project, grant number 248502. The views in this paper are those of the authors.

\section{References}

1. Atkinson, K., Bench-Capon, T., Cartwright, D., Wyner, A.: Semantic models for policy deliberation. In: Proceedings of the Thirteenth International Conference on Artificial Intelligence and Law (ICAIL 2011). pp. 81-90. Pittsburgh, PA, USA (2011)

2. Atkinson, K., Bench-Capon, T.J.M.: Abstract argumentation scheme frameworks. In: Dochev, D., Pistore, M., Traverso, P. (eds.) AIMSA. Lecture Notes in Computer Science, vol. 5253, pp. 220-234. Springer (2008)

3. Barabasi, A.L., Oltvai, Z.N.: Network biology: understanding the cell's functional organization. Nature Reviews Genetics 5(2), 101-113 (Feb 2004)

4. Barabási, A.L.L., Gulbahce, N., Loscalzo, J.: Network medicine: a network-based approach to human disease. Nature reviews. Genetics 12(1), 56-68 (Jan 2011)

5. Black, E., Atkinson, K.: Choosing persuasive arguments for action. In: Proceedings of the Tenth International Conference on Autonomous Agents and Multiagent Systems (AAMAS 2011) (2011)

6. Cerami, E.G., Gross, B.E., Demir, E., Rodchenkov, I., Babur, O., Anwar, N., Schultz, N., Bader, G.D., Sander, C.: Pathway commons, a web resource for biological pathway data. Nucleic Acids Research 39(suppl 1), D685-D690 (2011) 
7. Dung, P.M.: On the acceptability of arguments and its fundamental role in nonmonotonic reasoning, logic programming and n-person games. Artificial Intelligence $77(2), 321-358(1995)$

8. Dunne, P., Wooldridge, M.: Complexity of abstract argumentation. In: Rahwan, I., Simari, G. (eds.) Argumentation in Artificial Intelligence, pp. 85-104. Springer (2009)

9. Egly, U., Gaggl, S.A., Woltran, S.: Answer-set programming encodings for argumentation frameworks. Argument and Computation 1(2), 147-177 (2008)

10. Hermjakob, H., MontecchiPalazzi, L., Lewington, C., Mudali, S., Kerrien, S., Orchard, S., Vingron, M., Roechert, B., Roepstorff, P., Valencia, A., Margalit, H., Armstrong, J., Bairoch, A., Cesareni, G., Sherman, D., Apweiler, R.: Intact: an open source molecular interaction database. Nucleic Acids Research 32(suppl 1), D452-D455 (2004)

11. Hidalgo, C.A., Blumm, N., Barabsi, A.L., Christakis, N.A.: A dynamic network approach for the study of human phenotypes. PLoS Comput Biol 5(4), e1000353 (04 2009)

12. Jeong, H., Mason, S.P., Barabasi, A.L., Oltvai, Z.N.: Lethality and centrality in protein networks. Nature 411(6833), 41-42 (May 2001)

13. Kitano, H.: Computational systems biology. Nature 420(6912), 206-210 (November 2002)

14. Kitano, H.: Systems Biology: A Brief Overview. Science 295(5560), 1662-1664 (March 2002)

15. Kuhn, M., Campillos, M., Letunic, I., Jensen, L.J., Bork, P.: A side effect resource to capture phenotypic effects of drugs. Molecular Systems Biology 6(1) (Jan 2010)

16. McLeod, K., Ferguson, G., Burger, A.: Using argumentation to resolve conflict in biological databases. In: Green, N., Grasso, F., Kibble, R., Reed, C. (eds.) Proceedings of Computational Models of Natural Argument (CMNA) 9. pp. 15-23 (2009)

17. McLeod, K., Ferguson, G., Burger, A.: Argudas: arguing with gene expression information. In: Paschke, A., Burger, A., Splendiani, A., Marshall, M.S., Romano, P. (eds.) Proceedings of the 3rd International Workshop on Semantic Web Applications and Tools for the Life Sciences (December 2010)

18. McLeod, K., Burger, A.: Towards the use of argumentation in bioinformatics: a gene expression case study. In: ISMB. pp. 304-312 (2008)

19. Prakken, H.: An abstract framework for argumentation with structured arguments. Argument and Computation 1(2), 93-124 (2010)

20. Walton, D.: Argumentation Schemes for Presumptive Reasoning. Erlbaum, Mahwah, N.J. (1996)

21. Walton, D., Reed, C., Macagno, F.: Argumentation Schemes. Cambridge University Press (2008)

22. Wooldridge, M., van der Hoek, W.: On obligations and normative ability: Towards a logical analysis of the social contract. Journal of Applied Logic 3(3-4), 396-420 (2005)

23. Wyner, A., Bench-Capon, T., Atkinson, K.: Formalising argumentation about legal cases. In: Proceedings of the Thirteenth International Conference on Artificial Intelligence and Law (ICAIL 2011). pp. 1-10. Pittsburgh, PA, USA (2011)

24. Zhao, J., Gong, A.Y., Zhou, R., Liu, J., Eischeid, A.N., Chen, X.M.: Downregulation of pcaf by miR-181a/b provides feedback regulation to tnv- $\alpha$-induced transcription of proinflammatory genes in liver epithelial cells. The Journal of Immunology (2012) 